\title{
EXPERIMENTAL INVESTIGATION ON WIND ENERGY HARVESTING BY AEROELASTIC EXCITATION OF A SEMI-CIRCULAR CYLINDER WITH FLAT SIDE PARALLEL TO THE INCOMING FLOW
}

\author{
MARINA G. LÓPEZ-ARIAS, FELIX NIETO \& SANTIAGO HERNÁNDEZ \\ School of Civil Engineering, University of La Coruna, Spain.
}

\begin{abstract}
In this work, the performance of a semicircular cylinder with the flat side parallel to the incoming flow has been studied experimentally in a wind-excited energy harvester. Preliminary sectional model wind tunnel tests have allowed the identification of the reduced velocity ranges showing appreciable wind-induced oscillations. Interestingly, vortex-induced vibration-galloping interferences have been identified for both heave and pitch degrees of freedom at low reduced wind speeds, as well as coupled galloping in the higher ranges of flow velocities. The impact on the aeroelastic response caused by the attachment of a small-diameter rod at different positions has been studied, finding an enhancement in the amplitude of the oscillations and an enlargement in the vortex-induced vibration excitation range for some of them. Based on these preliminary results, an energy harvester has been designed adopting a cantilever beam supporting the semicircular cylinder. The device has showed a good performance reaching $2.5 \mathrm{~mW}$ of averaged harvested power for the configuration with a small-diameter rod attached at $\gamma=85^{\circ}$ for $8 \mathrm{~m} / \mathrm{s}$ flow speed.
\end{abstract}

Keywords: Energy harvesting, Galloping, Piezoelectricity, Semi-circular cylinder, Vortex-induced vibration, Wind tunnel.

\section{INTRODUCTION}

Climate change and its consequences have been an increasingly worrisome issue these last decades. The quest for renewable energy generation systems as a replacement for fossil fuels has triggered research efforts in order to find an efficient solution to this well-known problem. Consequently, a decarbonisation process aiming at replacing non-renewable fossil fuels by renewable energy sources is underway, showing an increasing contribution of the latter in the energy mix [1]. This process has been gaining momentum thanks to the decisive policies led by multinational bodies such as the European Green Deal or the UN's Sustainable Development Goals.

In the natural environment, several energy sources are available, which are therefore renewable, such as solar, wind, wave, marine or thermal. Fluid flows, such as a wind or tidal currents, contain huge amounts of kinetic energy. In fact, humankind has used wind and water flows to power mills or pump water for centuries [2]. Focusing on wind energy, the use of wind turbines is widespread. In recent years, there has been a boom in the technological development of these devices aiming at offering a feasible solution for power generation in different environments. Hence, vertical-axis wind turbines (VAWT) have been developed, typically for high-turbulence environments, while the more common horizontal-axis wind turbines (HAWT) have been enlarged and fine-tuned for high-scale generation in the framework of both onshore and offshore deployments.

However, there has been also a growing need for small-scale energy generation to be used for powering small electronic devices [3] such as monitoring systems or medical implants as well as in military infrastructures and wireless systems in smart cities [4]. The kinetic energy 
contained in a flow can be transformed into mechanical energy when a body immersed in the flow is excited due to the aeroelastic interaction between the flow and the body. The aeroelastic phenomena that may take place are ample depending on the dynamic characteristics of the body, the angle of attack between the body and the flow, the flow mean speed and its turbulent characteristics. Typical phenomena are flutter, galloping, vortex-induced vibration, buffeting or wake galloping [2].

The main goal of this work was to understand the aeroelastic phenomena that the semi-circular cylinder experiences in order to develop an energy harvester prototype, and study its performance in terms of displacement, voltage output and harvested power. For this purpose, a series of sectional model wind tunnel tests were carried out first. Afterwards, the full prototype performance was tested in the wind tunnel. Preliminary results of this study have been reported in [5].

The organization of this paper is as follows: in section 2 the energy harvester concept is introduced, while in section 3 the aeroelastic phenomena fundamentals and basic formulation are described. Afterwards, the preliminary sectional model tests are introduced and the results obtained reported. In the following section, the design and experimental campaign conducted for an energy harvester prototype based on the results of the preliminary tests is reviewed. Finally, the main conclusions extracted from this piece of research are summarized and future lines of research are outlined.

\section{ENERGY HARVESTING SYSTEMS}

Energy harvesting studies how environmental excitations may be efficiently transformed into electrical power. According to Elvin and Erturk [6], energy harvesters convert mechanical into electrical energy by using many methods or principles, such as piezoelectric, electromagnetic or electrostatic. Piezoelectric energy harvesting tends to be the most common method due to advantages in application, fabrication and higher voltage output [7]. The most frequent arrangement for wind-excited energy harvesters consists of a flexible cantilever beam with piezoceramic layers attached near the base and a bluff body fixed at the free end of the cantilever.

The piezoelectric principle is present only in certain materials with a symmetric crystalline structure. When the material is subject to no stress, the ions that the matter structure is composed of remain symmetrically distributed, and no polarization takes place. However, when the material is under stress, the matter structure experiences a small shift of the body centre site cations, resulting in a polarization of the atom that gives place to an electrical field in the material. This is called the direct effect of the piezoelectric material and can be reversed if an electrical field is applied, resulting in a strain in the material.

It is crucial that the mechanical energy that is required for the piezoelectric effect to take place originates from a renewable energy source such as the wind. Therefore, wind-induced oscillations from the aeroelastic excitation of bluff bodies have been studied in order to obtain an adequate amount of mechanical energy to be converted into electrical energy. These wind-induced oscillations are generated by the changes in the pressure around an object submerged in a fluid flow, such as the wind. This mutual interaction between the flow and the body is known as aeroelasticity, and its nature depends on variables such as the body's geometry, flow velocity and the angle of attack, to name a few. The main phenomena that this work has focused on are vortex-induced vibration (VIV), and galloping.

The aim of piezoelectric energy harvesting from wind-induced oscillations is the use of both piezoelectric materials and wind power to obtain a sufficiently large amount of electrical 
energy for low-powered devices, such as sensors. For this purpose, energy harvesters are designed to enhance wind-induced oscillations resulting from aeroelastic phenomena.

Several research works have been published these recent years regarding piezoelectric energy harvesting from aeroelastic phenomena, such as VIV and galloping. Focusing on experimental approaches, Hu et al. [8] studied the performance of an energy harvester prototype excited by the VIV of a square prism attached to the tip of an aluminium cantilever beam. Wind tunnel tests were carried out with the purpose of comparing the results of the basic configuration and an alternative one considering the attachment of fins to the prism's corners to enhance its aeroelastic response. Another relevant experimental work on this subject was carried out by Elvin and Elturk [6]. In their work, a piezoelectric energy harvester consisting of a cantilever beam with a tip body was tested in the wind tunnel. Three different geometries for the tip body were studied: a circular cylinder, a semi-circular cylinder with the flat side orthogonal to the incoming flow, and a flat plate. Results from this study showed that the use of a semi-circular cylinder significantly increased the harvested power. Andrianne at al. [9] also published an experimental work focusing on electromagnetic energy harvesters excited by VIV and VIV-galloping interaction of a square cylinder. Furthermore, the parametric space studied experimentally was extended in the aforementioned reference, introducing coupled electro-aeroelastic models. The outcome of their work has resulted in the identification of a better performance in terms of power output for the VIV-galloping interaction configuration.

On the other hand, studies approaching energy harvesting based on CFD simulations have been scarce. For instance, Abdelkefi et al. [10] studied piezoelectric energy harvesters excited by vortex-induced vibrations of circular cylinders. In their research, numerical simulations were performed to study the effect of the load resistance, as a circuit parameter of the piezoelectric transducer, on the oscillation amplitude, lift coefficient, voltage output and harvested power. In another publication, $\mathrm{Hu}$ et al. [11] discussed the performance of a wind energy harvester composed of circular cylinders in a tandem arrangement and compared power output results when protuberances were added to the cylinders, via CFD. The main conclusion from this work was that the energy harvested with added protrusions presented a better performance for a high wind velocity environment, whereas the plain cylinders worked more efficiently for low wind velocities. Another numerical study published by Ding et al. [12] studied wind energy harvesting from bluff bodies with different cross-sections. Results from this study suggested that the optimum regime for energy harvesting applications is the VIV upper branch, where maximum efficiencies were reached.

In our work, the focus has been on a semi-circular body geometry, also known as the $\mathrm{D}$-section, at $\mathrm{a}=0^{\circ}$ angle of attack (flat side parallel to the incoming flow). This geometry is of growing interest due to its increasing use in construction. Despite this fact, not many aerodynamic studies have been published regarding this cross-section. Den Hartog [13] was the first to study this geometry's galloping instabilities, while Novak and Tanaka [14] reported the effects on the galloping instability and force coefficients caused by smooth and turbulent flows. Cid Montoya et al. [15] addressed the semi-circular cylinder force coefficients and Strouhal number depending on the Reynolds number and the angle of attack. However, no significant study has been published regarding piezoelectric energy harvesting from aeroelastic phenomena present in the semi-circular cylinder at the angles of attack reported herein.

\section{AEROELASTIC PHENOMENA}

Among the many aeroelastic instabilities that may take place when a flow encounters a bluff body, VIV, galloping and flutter are the most common. This work principally focuses on the 
VIV and galloping responses of a semi-circular cylinder with the flat side parallel to the incoming flow. The main aerodynamic parameters that are used to describe these phenomena are presented below.

\subsection{Force coefficients and Strouhal number}

The force coefficients provide the non-dimensional value of the mean flow-induced loads acting on a static body, and are defined as:

$$
C_{D}=\frac{F_{D}}{\frac{1}{2} \rho U^{2} A} ; C_{L}=\frac{F_{L}}{\frac{1}{2} \rho U^{2} A} ; C_{M}=\frac{M}{\frac{1}{2} \rho U^{2} B A} ;
$$

where $F_{D}$ and $F_{L}$ are the drag and lift forces, $M$ is the moment, $\rho$ is the fluid density, $U$ the reference flow velocity, $A$ is the reference area and $B$ the reference length.

The Strouhal number represents the non-dimensional frequency at which vortices are shed into the wake of the body:

$$
S t=\frac{f D}{U},
$$

where $f$ is the frequency at which vortices are shed and $D$ is the reference across-wind length of the body. This number is characteristic of each body's geometry and depends on the Reynolds number, the angle of attack and the turbulent characteristics of the flow.

These non-dimensional parameters represent the aerodynamic characteristics of the bluff body; therefore, their values are crucial to understand the aeroelastic phenomena that may take place.

\subsection{Vortex-induced vibration}

Vortex-induced vibrations (VIV) is a phenomenon caused by the coupling of one of the bluff body's natural frequencies and the frequency at which vortices are shed from the body. When this occurs, the amplitude of oscillation of the body increases and the frequency of oscillation remains constant throughout a certain range of velocities, in a phenomenon known as lock-in [16].

The Strouhal number represents the non-dimensional frequency at which vortices are shed from the bluff body and shows a constant dependency on the excitation frequencies of the body and the flow velocity. This parameter is of utmost importance since it can be used to predict the flow velocity at which vortex-induced vibrations may appear.

Once the natural frequencies of the body are known, the flow velocity at which the vortex-shedding frequency matches those natural frequencies can be obtained through the Strouhal relation:

$$
U_{c, V I V}=\frac{f_{n} D}{S t}
$$

where $f_{n}$ is a natural frequency of the dynamical system, $D$ is the reference length and $S t$ is the Strouhal number that can be measured through aerodynamic wind tunnel tests for each bluff body.

In this work, values of the critical Strouhal number for the semi-circular cross-section were taken from Montoya et al. [15], and the natural frequencies of the body were measured in the wind tunnel facility to identify the critical VIV flow speed for the sectional models and prototype. 


\subsection{Galloping}

Galloping corresponds to an aeroelastic instability caused by a negative aerodynamic damping being introduced in the dynamical system. This phenomenon is usually characterised by large across-wind oscillations. The dynamic equilibrium equation of the system can be expressed as

$$
m\left(\ddot{y}+2 \zeta \omega_{n} \dot{y}+\omega_{n}^{2} y\right)=-\frac{1}{2} \rho U^{2} D\left(C_{D}+\frac{d\left(C_{L}\right)}{d a}\right) \frac{\dot{y}}{U},
$$

where $y$ is the across-wind displacement of the body, $m$ is the mass per unit length, $\zeta$ is the mechanical damping ratio, $\omega_{n}$ is the natural circular frequency of oscillation and $d / d \alpha$ is the derivative with respect to the flow angle of attack $\alpha$ (see Figure 1). Given equation (4), the sufficient condition for the galloping instability is

$$
\left(C_{D}+\frac{d\left(C_{L}\right)}{d \alpha}\right)_{a=0}<\frac{-4 m \zeta \omega_{n}}{\rho U D}=\frac{-2 c}{\rho U D} .
$$

Hence, a necessary condition for galloping is

$$
\left(C_{D}+\frac{d\left(C_{L}\right)}{d a}\right)_{a=0}<0
$$

which is known as the Den Hartog criterion [16], where the left-hand term will hereafter be referred to as $a_{g}$. This parameter depends on the bluff body's geometry and the angle of attack and is obtained from measured force coefficients. The critical wind speed for galloping to occur can be obtained as:

$$
U_{c, \text { galloping }}=S c \cdot \frac{n D}{-a_{g}},
$$

where $S c$ is the Scruton number, indicative of the mass-damping ratio in the system, and $n$ refers to the natural frequency of oscillation of the dynamical system.

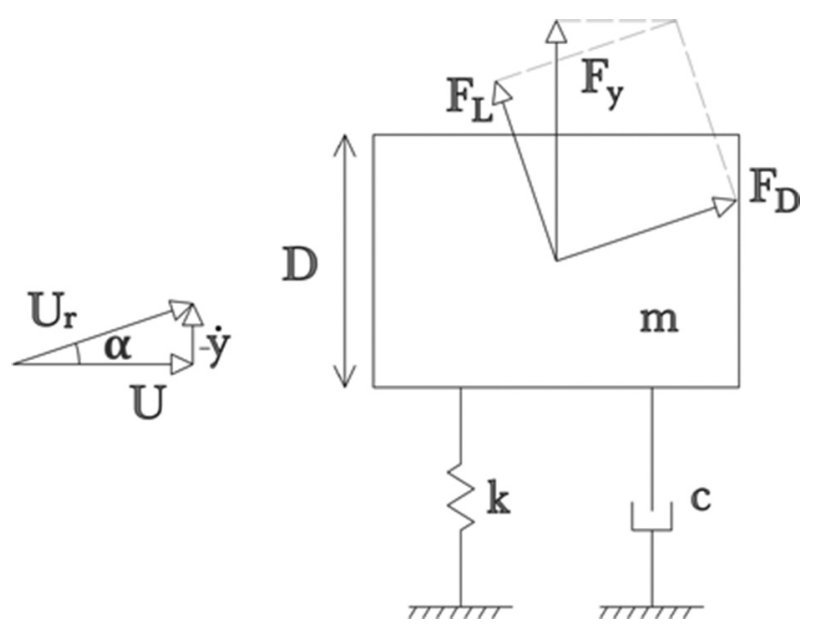

Figure 1: Galloping model: single degree of freedom system under flow-induced forces. 
It is important to mention that for $a_{g}$ values close to zero, the critical wind speed for galloping presents large variations for a small change in the $a_{g}$ parameter. This issue leads to a poor prediction of the galloping critical wind speed for low values of $a_{g}$. Many authors, such as Mannini et al. [17], have reported this variability of the parameter $a_{g}$ to be highly dependent on how the force coefficients have been fitted and how the estimation of the lift coefficient derivative has been made.

\section{EXPERIMENTAL IMPLEMENTATION}

A series of free-to-oscillate wind tunnel tests were carried out in order to characterise the aeroelastic performance of the semi-circular geometry considering the flat side parallel to the incoming wind flow direction. The main goal of these tests was to point out the ranges of reduced wind velocities at which significant oscillations of the bluff body appear, as well as characterising the aeroelastic phenomena taking place. All the tests presented in this paper were performed at the Aerodynamic Wind Tunnel (TUVA) of the University of A Coruna, Spain. This is an open circuit wind tunnel with a square test section of 1 by $1 \mathrm{~m}^{2}$. The contraction rate in the nozzle is $6: 1$, while the maximum flow speed is $28 \mathrm{~m} / \mathrm{s}$. The turbulent intensity at the inlet of the test chamber is $2 \%$.

Figure 2 shows a scheme for the supporting system of the sectional model in the wind tunnel tests that were performed for the preliminary campaign. The sectional model was supported by load cells through a series of springs. The $5 \mathrm{~kg}$ Utilcell Mod. 240 load cells have an accuracy of $0.6 \mathrm{~g}$. In these tests, as the component of interest is the across-wind response, the along-wind motion depending on $k_{H}$, the stiffness of the horizontal springs in Figure 2, has been constrained using stiffer springs.

Damping and natural frequency values have been obtained for this configuration, resulting in a damping ratio relative to critical of the dynamical system in the across-wind degree of freedom of $\xi=0.0014$. In Table 1 , the measured natural frequencies of the dynamical system are presented. It should be noted that the across-wind and the torsional degrees of freedom natural frequencies were relatively close, suggesting a potential for coupled galloping. For this purpose, accelerometers Brüel \& Kjaer 4508-B-001 have been employed, with a sensitivity of $1 \mathrm{mV} / \mathrm{ms}-2$.

Critical wind velocities for both VIV and galloping have been obtained using the values in Table 1 and data in the literature. First, through the Strouhal relation, the Karman-vortex

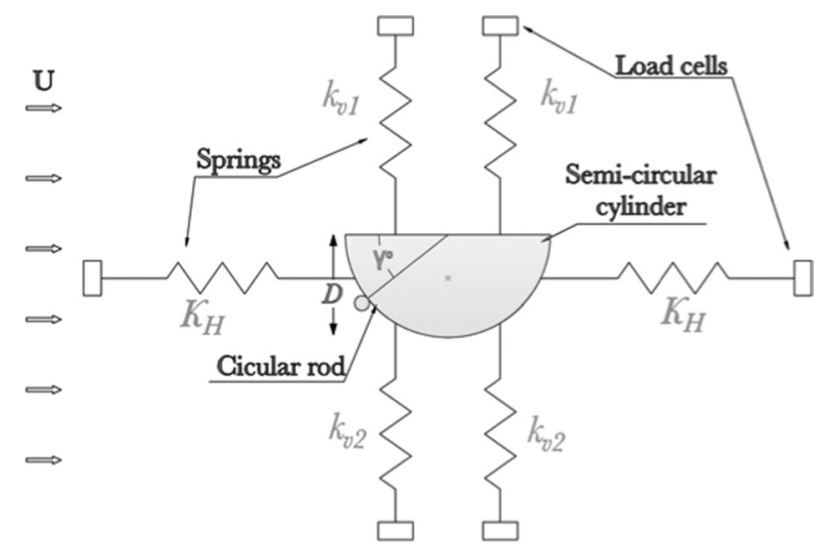

Figure 2: Sectional model test configuration of the preliminary wind tunnel tests ( $D$ represents the depth of the body). 
Table 1: Measured natural frequencies of the semi-circular sectional model.

\begin{tabular}{ll}
\hline Degree of freedom & Natural frequency $(\mathbf{H z})$ \\
\hline Along-wind & 3.52 \\
Across-wind & 1.56 \\
Torsional & 1.86 \\
\hline
\end{tabular}

Table 2: Critical flow speed for VIV for the sectional model tests.

\begin{tabular}{ll}
\hline Degree of freedom & $(\mathbf{m} / \mathbf{s})$ \\
\hline Across-wind & 1.28 \\
Torsional & 1.89 \\
\hline
\end{tabular}

resonance flow speed, Equation (3), at which the frequency of the vortex-shedding matches one of the natural frequencies of the model, was identified. The Strouhal number was taken as $S t=0.207$ from Cid Montoya et al. [15] and the depth of the body, $D$, was taken as the cylinder radius, with a value of $D=0.17 \mathrm{~m}$. Results for the critical Karman vortex resonance flow speed are presented in Table 2.

On the other hand, the critical wind speed for galloping instability has been also identified, and for this matter, the $a_{g}$ parameter has been obtained. This parameter, introduced in section 3.3, corresponds to the sum of the drag coefficient and the first derivative of the lift coefficient with respect to the angle of attack. Data for the force coefficients of the semi-circular body were taken from Cid Montoya et al. [15]. In order to obtain the $a_{g}$ parameter, a linear estimation of the derivative of the lift coefficient was made, obtaining the value shown in Table 3. As the final value of $a_{g}$ was positive, the Den Hartog criterion was satisfied and therefore, the necessary condition for a galloping instability to occur is met. The Scruton number for the sectional model was taken as 1.28.

It should be noted that the reference sectional model is tested for $\alpha=0^{\circ}$ angle of attack; and later, a protuberance to the shape of the semi-circular cylinder was introduced adding a small rod attached at different positions.

\subsection{Wind tunnel tests for $\alpha=0^{\circ}$ angle of attack.}

According to the properties and parameters previously introduced, the wind tunnel tests of the sectional model were conducted for the $\alpha=0^{\circ}$ angle of attack. The wind speed inside the chamber was increased gradually, and the displacements obtained through the loads registered by the load cells supporting the body. Results were obtained for the across-wind degree of freedom, which will hereafter be referred to as heave, and for the torsional degree of freedom, referred to as pitch. It should be noted that, although the results have been reported separately for the heave and pitch degrees of freedom for the sake of clarity, the two degrees of freedom were free to oscillate simultaneously during the experiments.

Table 3: Critical flow speed for galloping and $a_{g}$ parameter for the sectional model tests.

\begin{tabular}{lll}
\hline Data taken from & $\boldsymbol{a}_{\boldsymbol{g}}$ & $\boldsymbol{U}_{\boldsymbol{c}, \text { galloping }}(\mathrm{m} / \mathrm{s})$ \\
\hline Cid Montoya et al. [15] & 1.23 & 1.04 \\
\hline
\end{tabular}




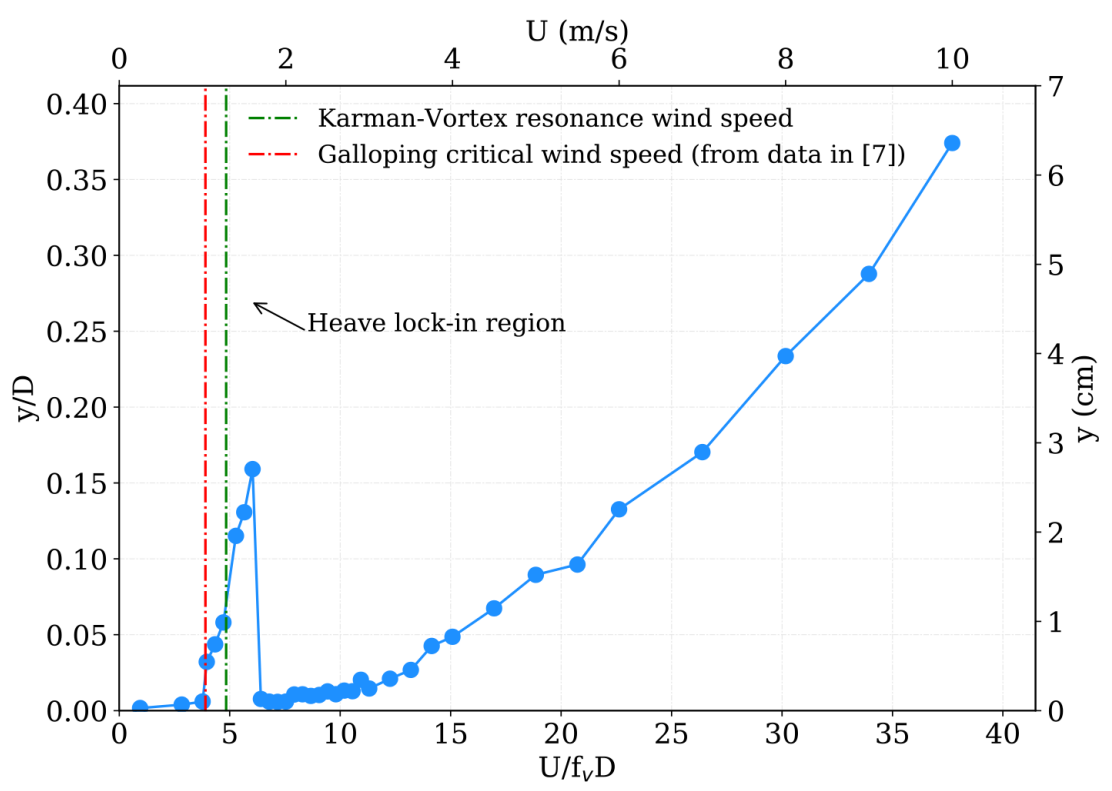

Figure 3: Heave motion for the semi-circular as function of the non-dimensional wind velocity.

Figure 3 shows the heave motion for the tests conducted at different flow velocities. The predicted critical wind speeds for both VIV and galloping are also plotted. It should be noted that the critical speed for galloping is lower than the Karman-vortex resonance wind speed, suggesting full VIV-galloping interference behaviour as has been described in Mannini et al. [18] for small Scruton numbers. From this figure, a "bell" shape can be identified between 1 $\mathrm{m} / \mathrm{s}$ and $2 \mathrm{~m} / \mathrm{s}$, with a peak non-dimensional displacement value of 0.17 , presenting a typical VIV behaviour. The asynchronous quenching effect, preventing the divergent instability at the galloping critical speed has been identified, starting the divergent branch at a flow speed higher than the critical speed for VIV due to the interaction with the pitch degree of freedom that is later described.

Therefore, an ample range of relatively low wind velocities has shown a potential for a significative wind-induced motion in the heave degree of freedom and, consequently energy harvesting.

For the pitch degree of freedom motion, results are presented in Figure 4. The VIV phenomenon has been also identified for wind velocities ranging from 2 to $3 \mathrm{~m} / \mathrm{s}$, which fits well with the predicted critical wind velocity for the torsional degree of freedom in Table 2. Subsequently, the pitching motion amplitude showed a slight decay and rapidly increased as the flow speed was augmented. This kink in the displacement-velocity curve at $U / U_{C, V I V}=1.5$ is similar to the results reported in Mannini et al. [17] for a 3:2 ratio rectangular cylinder and might be indicative of a partial interference between VIV and galloping, as described by Mannini et al. [18].

It was mentioned that the values of the natural frequencies for the heave and pitch degree of freedom in Table 1 were fairly close. This proximity could be indicative of the potential for the coupling of the two modes when excited by the wind action. In Figures 3 and 4, the motion-velocity curves show that for reduced velocities higher than 10 , the amplitude of oscillation rapidly grows for both degrees of freedom. In Figure 5, the main excitation frequencies for the heave and pitch degrees of freedom are presented as functions of the wind 


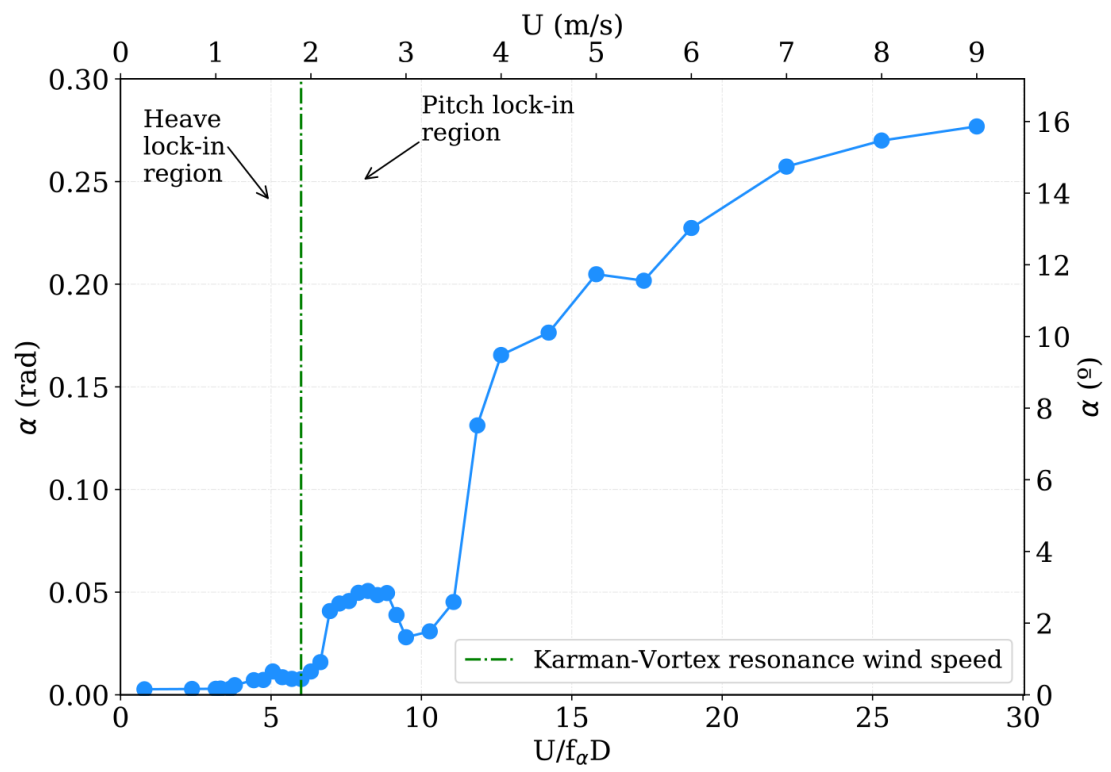

Figure 4: Pitch motion for the semi-circular cylinder as function of the non-dimensional wind velocity.

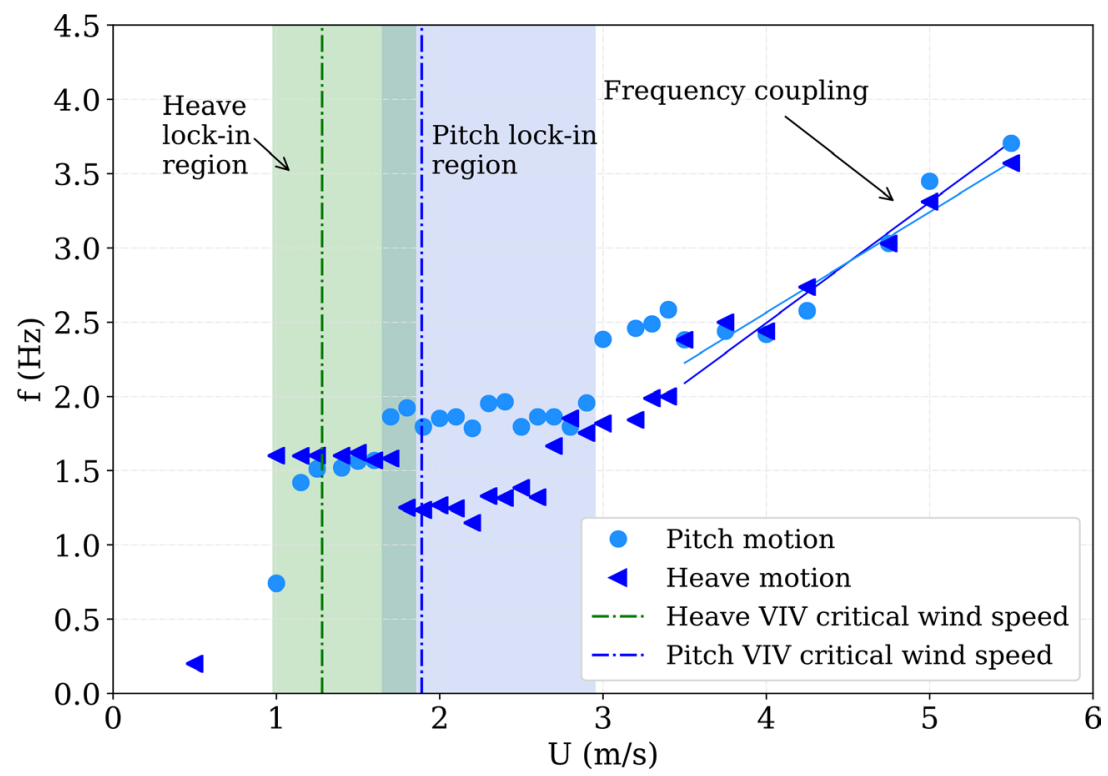

Figure 5: Heave and pitch main excitation frequencies vs flow speed at a $0^{\circ}$ angle of attack.

speed. In the chart, both the heave and the pitch lock-in regions can be identified as plateaus of nearly constant frequency. However, for flow speeds higher than $4 \mathrm{~m} / \mathrm{s}$, both degrees of freedom excitation frequencies approach the same values, indicating a coupled galloping excitation of the dynamical system. 


\subsection{Wind tunnel tests at $\alpha=0^{\circ}$ angle of attack with attached rod}

The range of wind velocities at which VIV appears for the semi-circular section is crucial due to its linkage with the energy harvesting potential. Because of this, one strategy that was previously adopted aiming at enlarging the lock-in range and the amplitude of the response consists of modifying the external shape of the body tip by attaching small-diameter cylinder rods or fins (Hu et al. [8,11]), modifying the flow separation points in the body. In the aforementioned references, it is demonstrated how attaching triangular protuberances or rods to the bluff body may enhance the velocity range and amplitude response of the aeroelastic excitation. Therefore, another set of wind tunnel tests were conducted attaching a circular rod to the semi-circular cylinder at different positions, at angles $\gamma=15^{\circ}, 30^{\circ}, 45^{\circ}, 55^{\circ}, 65^{\circ}, 75^{\circ}$ and $85^{\circ}$ relative to the horizontal direction as shown in figure 2 . The circular rod used for the study had a diameter of $1 \mathrm{~cm}$. The results are presented in Figure 6.

In Figure 6(a), it can be seen that the circular rod did not considerably enlarge the lock-in range nor the amplitude of oscillation for the considered range of positions. In fact, for an angle of $15^{\circ}$, the VIV response completely disappeared. On the contrary, in Figure 6(b), the results show the sensitivity of the aeroelastic response with the position of the rod. When the angle of the rod attachment is larger than $75^{\circ}$, both the lock-in range of wind velocities and the maximum amplitude of oscillation grow, showing a higher potential for energy harvesting. In fact, both the amplitude of the response and the lock-in reduced velocity range enlarged up to a $20 \%$ with respect to the bare semi-circular cylinder geometry. Thus, for the energy harvester prototype, the configuration including a small-diameter circular rod attached at a $\gamma=85^{\circ}$ angle will be also studied to analyse its impact on the prototype's performance.

\subsection{Energy harvester prototype design}

Based on the previously reported preliminary wind tunnel campaign, an energy harvester prototype was designed and tested in the wind tunnel in terms of displacement, voltage and power output. This energy harvester consists of a cantilever steel beam whose deflection is generated by the wind-induced oscillations of the semi-circular cylinder located at its tip. Close to the fixed end of the cantilever beam, a Macro-Fiber Composite (MFC) piezoelectric sheet is bonded, and it acts as the transducer to convert the mechanical energy into electrical power. A scheme of the designed harvester is shown in Figure 7(a) and 7(b), with and without

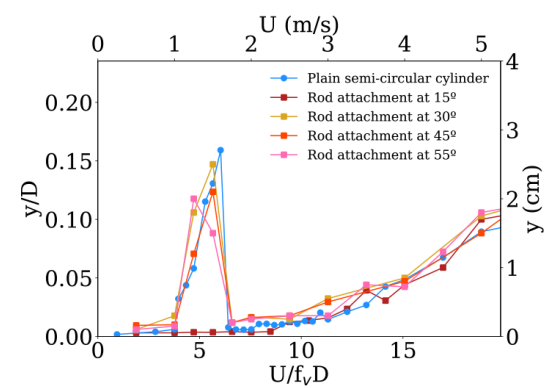

(a)

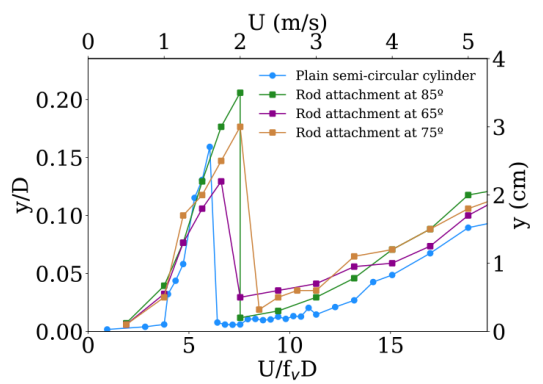

(b)

Figure 6: Non-dimensional amplitude of oscillation as function of the wind velocity for the attached circular rod wind tunnel campaign. (a) Results for $15^{\circ}$ to $55^{\circ}$ angle attachments; (b) Results for $65^{\circ}$ to $85^{\circ}$ angle attachments. 


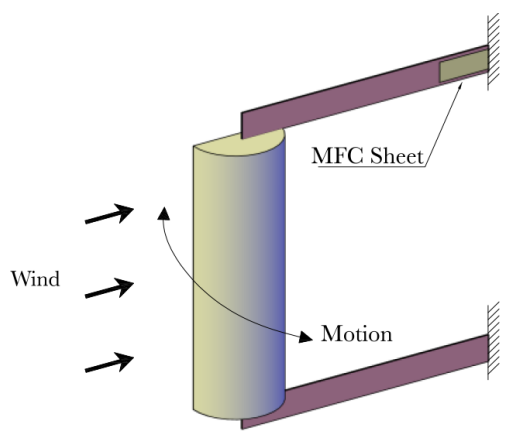

(a)

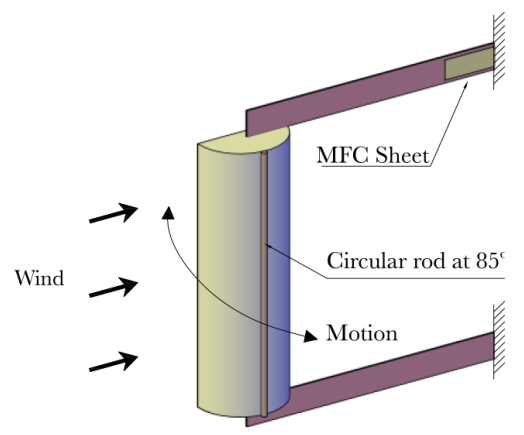

(b)

Figure 7: Energy harvester configurations used in this study. (a) Plain semi-circular cylinder; (b) Semi-circular cylinder with a circular rod attachment at $85^{\circ}$.

the circular rod attachment. An image of the prototype mounted in the test chamber is also presented in Figure 8.

The harvester was designed to provide a dynamic response similar to the one obtained in the preliminary sectional model tests. For this purpose, in Table 4, its dynamic properties are

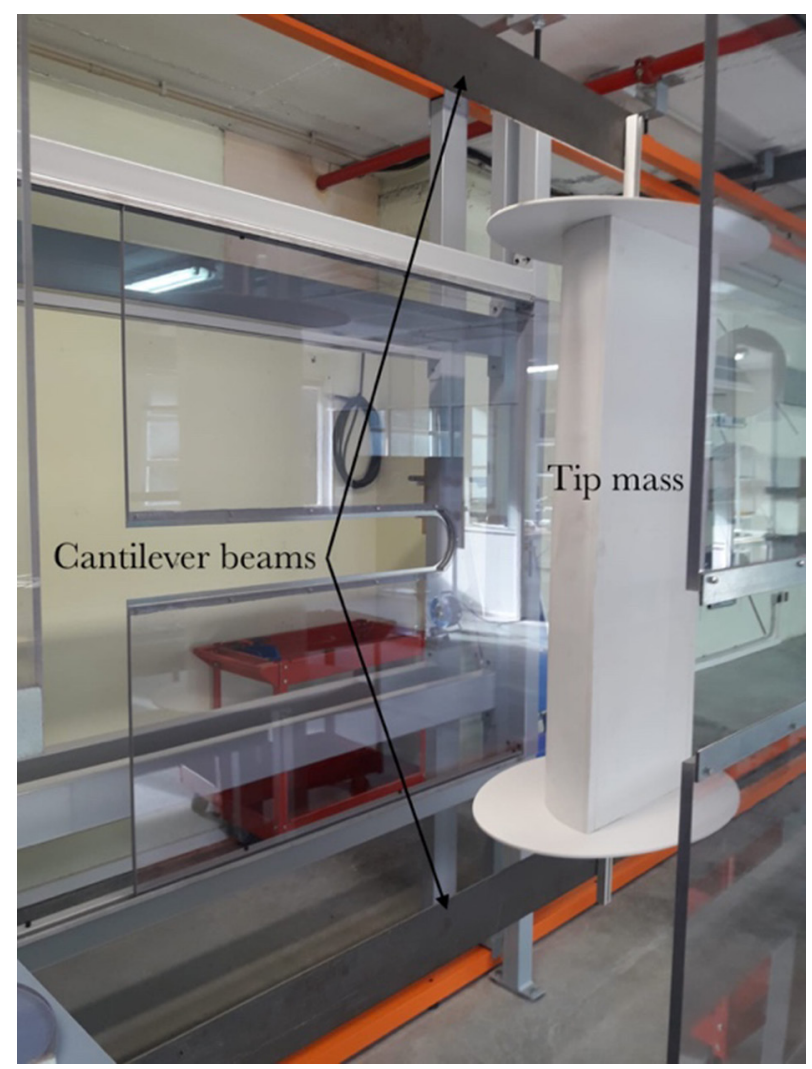

Figure 8: Energy harvesting prototype mounted in the test chamber. 
Table 4: Dynamic properties of the prototype.

\begin{tabular}{ll}
\hline Property & Value \\
\hline $1^{\text {st }}$ natural frequency & $1.56 \mathrm{~Hz}$ \\
Damping ratio & 0.011 \\
Mass & $5.63 \mathrm{~kg}$ \\
\hline
\end{tabular}

presented, where it can be seen how the first natural frequency of the system is close to the across-wind natural frequency of the previous tests. Structural analyses have been carried out in order to ensure the structural safety of the prototype.

In order to obtain the voltage output that the piezoelectric sheet generates, a measuring circuit was developed, based on an Arduino ONE board. In this study, the Arduino ONE board is connected to a PC for data acquisition. The Arduino ONE accuracy depends on its microcontroller, which has a \pm 2 least significant bits absolute accuracy. The board has also a voltage bound ranging from 0 to $5 \mathrm{~V}$, meaning that the measured voltage has to be in that range for it to be readable. However, the predicted voltage of the harvester has a sinusoidal wave shape [19] and is expected to be outside this range. Therefore, a voltage divider was used in the circuit, and modified throughout the study, to adjust the voltage peaks that the board receives, resulting in a voltage output of the highest measured value up to 10 times smaller than the input. In order to measure the full-length voltage wave, an offset of $1.5 \mathrm{~V}$ was introduced in the circuit, enough for the negative part of the wave to be above the null voltage value.

\section{RESULTS}

\subsection{Prototype wind tunnel tests results: voltage output}

As previously mentioned, the voltage generated by the MFC sheet has been measured and recorded by an Arduino board and a PC. It should be noticed that the semi-circular cylinder is rigidly connected to the tip of the beam, therefore the oscillations in the harvester were taking place in the first natural mode of the system, and no coupling between the heave and pitch degrees of freedom took place, as reported for the sectional model tests previously in Section 4.

The voltage output results presented herein are RMS voltage values, which are more representative than the voltage peak value. RMS stands for root mean square and represents the arithmetic mean of the squares of a set of values. Since the voltage response provided by the harvester is sinusoidal and the current is AC, the RMS voltage is equal to the value of the DC current that would provide the same amount of average power dissipation in a resistive load, which gives a better reference for the harvester response.

Sinusoidal waves for each sampled flow speed were obtained and the RMS values for the range of reduced wind velocities considered are presented in Figure 9. The results provided correspond to the energy harvester with the plain semi-circular cylinder as the tip body (Figure 7(a) configuration) and with the added circular rod (Figure 7(b) configuration). The voltage time history for a wind speed inside the lock-in region of $0.9 \mathrm{~m} / \mathrm{s}$ for the $0^{\circ}$ angle of attack test is also given in the graph.

It can be seen from Figure 9 that the prototype presents a narrow region of reduced velocities between 3 and 6 , where voltage grows due to the VIV phenomenon. This is seen for both the plain semi-circular prototype and the added rod prototype, although for this last 


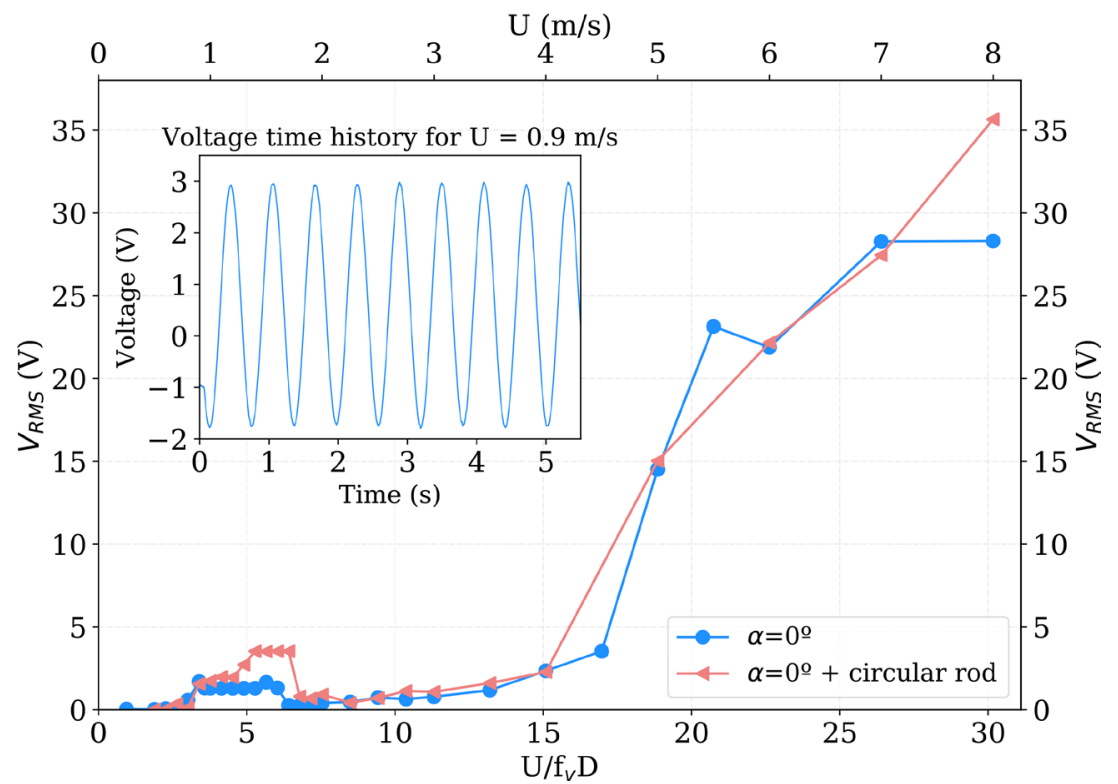

Figure 9: RMS Voltage output for the energy harvester with and without the circular rod attachment.

configuration the voltage has reached a peak RMS value of almost $5 \mathrm{~V}$, and encompassed a larger range of wind velocities. Nonetheless, for flow reduced velocities higher than 15 , the RMS voltage increased significantly, reaching a maximum of $28 \mathrm{~V}$ for the plain semi-circular prototype and of $36 \mathrm{~V}$ for the added rod prototype. The increase in the voltage output was due to an increase in the amplitude of oscillations, which can be associated to the built-up of galloping oscillations. It should be noted that the damping ratio of the prototype is one order of magnitude higher than in the sectional model, thus the Scruton number is larger, preventing the VIV-galloping interference.

\subsection{Wind tunnel tests results: power output}

The average harvested power that the prototype was able to generate at $0^{\circ}$ angle of attack with and without the added rod is presented in Figure 10. The average power is obtained from:

$$
P_{a v g}=\frac{V_{R M S}^{2}}{R},
$$

where $R$ is the electrical resistance and $V_{R M S}$ is the root mean square value of the voltage time history for a certain wind velocity.

Two values of electrical resistance were used in the final tests. The plain semi-circular cylinder harvester tests were performed using a value of $R=0.23 \mathrm{M} \Omega$ in the range of wind speeds lower than $4.5 \mathrm{~m} / \mathrm{s}$, while $R=0.5 \mathrm{M} \Omega$ was adopted for the higher range of wind speeds. It can be seen in Figure 10(a) that at the VIV wind velocity range, the maximum average power was about $12 \mu \mathrm{W}$. However, for reduced wind speeds higher than 20, presented in Figure 10(b), at the incipient galloping condition, the average power reached a value of $1700 \mu \mathrm{W}$. 


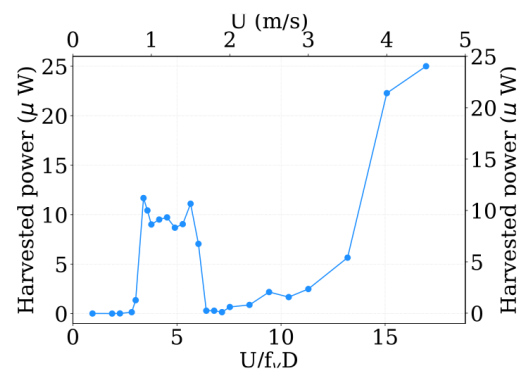

(a)

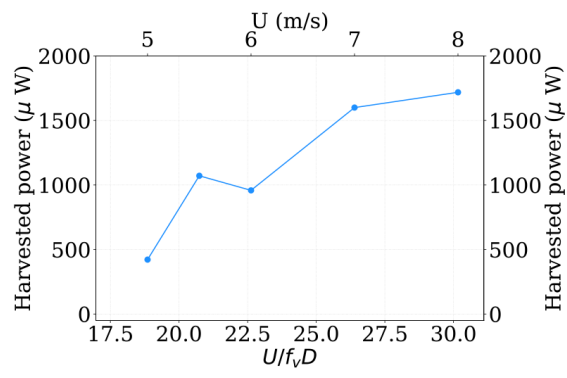

(b)

Figure 10: Average harvested power vs reduced wind speed for the plain semi-circular cylinder. (a) $U \leq 4.5 \mathrm{~m} / \mathrm{s}$ and $R=0.23 \mathrm{M} \Omega$; (b) $U>4.5 \mathrm{~m} / \mathrm{s}$ and $R=0.5 \mathrm{M} \Omega$.

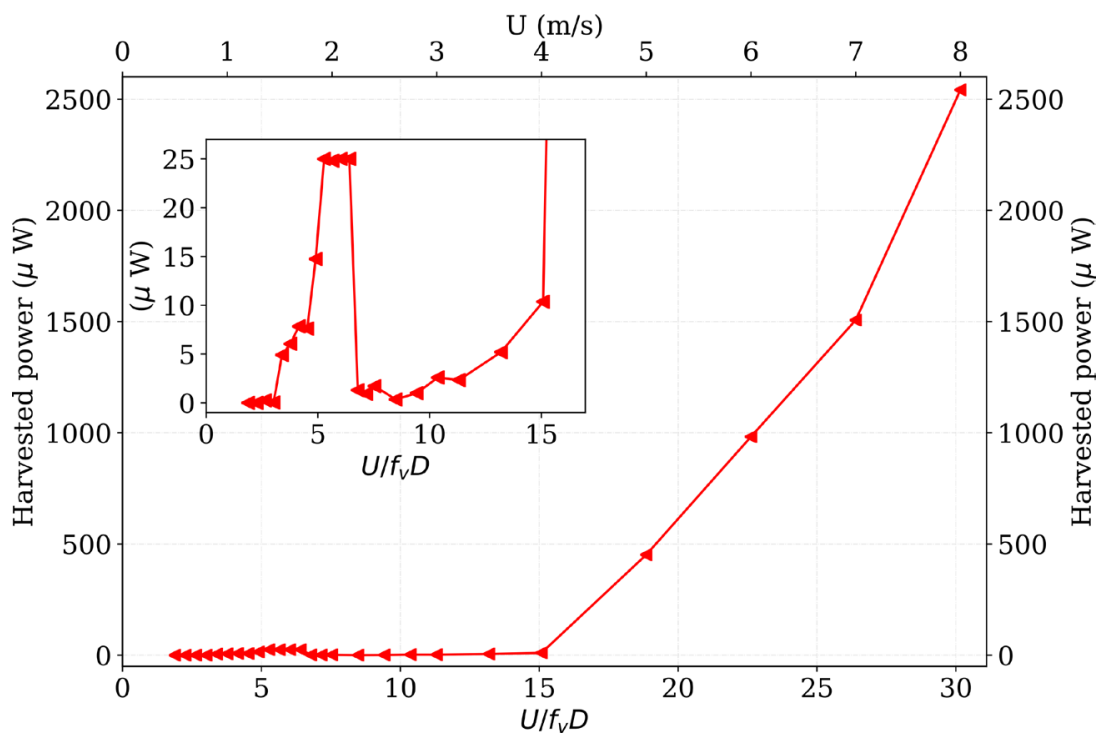

Figure 11: Average harvested power vs reduced wind speed for the semi-circular cylinder with the added rod with $R=0.5 \mathrm{M} \Omega$.

In Figure 11, power output results are provided for the harvester configuration with the attached rod. The resistance value used for this test is $R=0.5 \mathrm{M} \Omega$. Results have shown a maximum value of $25 \mathrm{~mW}$ for the low range of wind speeds, at the lock-in region. The absolute maximum average power has been reached at the incipient galloping condition, with a value of $2700 \mu \mathrm{W}$ at $U=8 \mathrm{~m} / \mathrm{s}$.

\section{CONCLUSIONS AND FUTURE WORK}

In this study, a piezoelectric energy harvester based on wind-induced oscillations of a bluff body was designed and studied experimentally for smooth flow at $0^{\circ}$ angle of attack. A circular rod was attached to the bluff body to enhance the prototype's performance and was also tested in the wind tunnel.

A series of preliminary wind tunnel tests of a sectional model of a semi-circular cylinder were first carried out. VIV-galloping interference in the heave and pitch degrees of freedom 
and coupled galloping were identified in this study. The model with the attached protuberance showed a larger VIV range and a higher maximum amplitude of oscillation, showing the ability of this approach to enhance wind-induced excitation.

The energy harvester prototype was designed based on the preliminary sectional model wind tunnel campaign. The results were obtained in terms of the voltage output generated by the piezoelectric sheet and the average harvester power, for each considered reduced wind velocity. The results have shown that both configurations presented VIV and galloping for the heave degree of freedom. However, the prototype with the circular rod attached as a protuberance offered higher values of both voltage and harvested power.

Based on the results obtained in this work, further research in this area may include the introduction of slight changes in the geometry of the bluff body that might improve the harvester's performance, the study of different angles of attack that may show different aeroelastic behaviour and the analysis of the impact in the electrical generation of different levels of turbulence in the flow, similar to those in natural wind.

\section{REFERENCES}

[1] Fanchi, J.R. \& Fanchi, C.J., Energy in the 21st Century, 4th ed., World Scientific Publishing, 2017.

[2] Rostami, A.B. \& Armandei, M., Renewable energy harvesting by vortex-induced motions: Review and benchmarking of technologies. Renewable and Sustainable Energy Reviews, 70, pp. 193-214, 2017. https://doi.org/10.1016/j.rser.2016.11.202

[3] Akaydin, H.D., Elvin, N. \& Andreopoulos, Y., Flow-induced vibrations for piezoelectric energy harvesting. Advances in Energy Harvesting Methods, Springer: New York, NY, pp. 241-267, 2013.

[4] Beeby, S.P., Tudor, M.J. \& White, N.M., Energy harvesting vibration sources for microsystems applications. Measurement Science and Technology, 17, pp. R175-R195, 2006. https://doi.org/10.1088/0957-0233/17/12/r01

[5] López-Arias, M.G., Nieto, F. \& Hernández, S., Experimental study for wind energy harvesting based on the aeroelastic excitation of a semi-circular cylinder. WIT Transactions on Engineering Sciences, Vol. 128, pp. 139-149, 2020. WIT Press, ISSN 17433533 (on-line).

[6] Elvin, N. \& Erturk, A., Introduction and methods of mechanical energy harvesting. Advances in Energy Harvesting Methods, Springer: New York, NY, pp. 3-14, 2013.

[7] Arroyo, E., Badel, A., Formosa, F., Wu, Y. \& Qiu, J. Comparison of electromagnetic and piezoelectric vibration energy harvesters: Model and experiments. Sensors and Actuators A: Physical, 183, pp. 148-156, 2012. https://doi.org/10.1016/j.sna.2012.04.033

[8] Hu, G., Tse, K.T., \& Kwok, K.C., Enhanced performance of wind energy harvester by aerodynamic treatment of a square prism. Applied Physics Letters, 108(12), 123901, 2016. https://doi.org/10.1063/1.4944555

[9] Andrianne, T., Aryoputro, R.P., Laurent, P., Colson, G., Amandolese, X. \& Hémon, P., Energy harvesting from different aeroelastic instabilities of a square cylinder. Journal of Wind Engineering and Industrial Aerodynamics, 172, pp. 164-169, 2018. https://doi. org/10.1016/j.jweia.2017.10.031

[10] Abdelkefi, A., Hajj, M.R. \& Nayfeh, A.H., Phenomena and modeling of piezoelectric energy harvesting from freely oscillating cylinders. Nonlinear Dynamics, 70(2), pp. 1377-1388, 2012. https://doi.org/10.1007/s11071-012-0540-x 
[11] Hu, G., Liu, F., Li, L., Li, C., Xiao, Y. \& Kwok, K.C.S., Wind energy harvesting performance of tandem circular cylinders with triangular protrusions. Journal of Fluids and Structures, 91, 102780, 2019. https://doi.org/10.1016/j.jfluidstructs.2019.102780

[12] Ding, L., Zhang, L., Wu, C., Mao, X. \& Jiang, D., Flow induced motion and energy harvesting of bluff bodies with different cross sections. Energy Conversion and Management, 91, pp. 416-426, 2015.

[13] Den Hartog, J.P., Transmission line vibration due to sleet. Transactions of the American Institute of Electrical Engineers, 51(4), pp. 1074-1076, 1932. https://doi.org/10.1109/taiee.1932.5056223

[14] Novak, M. \& Tanaka, H., Effect of turbulence on galloping instability. Journal of the Engineering Mechanics Division, ASCE, 100(1), pp. 27-47, 1974. https://doi.org/ 10.1061/jmcea3.0001861

[15] Cid Montoya, M., Nieto, F., Alvarez, A.J., Hernández, S., Jurado, J.A. \& Sánchez, R., Numerical simulations of the aerodynamic response of circular segments with different corner angles by means of 2D URANS. Impact of turbulence modeling approaches. Engineering Applications of Computational Fluid Mechanics, 12(1), pp. 750-779, 2018. https://doi.org/10.1080/19942060.2018.1520741

[16] Simiu, E. \& Scanlan, R.H., Wind Effects on Structures: Fundamentals and Applications to Design, 3rd ed., JohnWiley \& Sons, 1996.

[17] Mannini, C., Marra, A.M. \& Bartoli, G., VIV-galloping instability of rectangular cylinders: Review and new experiments. Journal of Wind Engineering and Industrial Aerodynamics, 132, pp. 109-124, 2014. https://doi.org/10.1016/j.jweia.2014.06.021

[18] Mannini, C., Marra, A.M., Massai, T. \& Bartoli, G., Interference of vortex-induced vibration and transverse galloping for a rectangular cylinder. Journal of Fluids and Structures, 66, pp. 403-423, 2016. https://doi.org/10.1016/j.jfluidstructs.2016.08.002

[19] Akaydin, H.D., Elvin, N. \& Andreopoulos, Y., Energy harvesting from highly unsteady fluid flows using piezoelectric materials. Journal of Intelligent Material Systems and Structures, 21(13), pp. 1263-1278, 2010. https://doi.org/10.1177/1045389x10366317 